\title{
Linear Coupling of Betatron Oscillations in the Main Ring
}

\author{
S. Ohnuma
}

February 9, 1978

$\underline{\text { Summary }}$

The linear coupling of betatron oscillations for $v_{\mathrm{H}}=v_{\mathrm{V}}$ is driven by the average skew quadrupole field in the ring. The purpose of this note is to point out that, since the driving term in the Hamiltonian is in general a complex quantity, a complete elimination of the coupling may require a correction skew quadrupole field with two independent parameters. Because of the long straight insertions which do not follow the FODO symmetry of the regular part of the lattice, the present correction scheme of the main ring, either with the correction skew quadrupoles at stations \#27, \#37 and \#43 or with the rolled regular $7^{\prime}$ quadrupoles at stations \#25 and \#43, cannot meet this requirement. It may be necessary to have correction skew quadrupoles at stations \#Il and \#49 and to roll quadrupoles in long straight sections. Numerical examples which demonstrate this are presented. 
I.

Since the emittance of the beam injected into the main ring is now practically indentical in two transverse directions, one may very well question the necessity of eliminating the linear couplings of the type $\nu_{\mathrm{H}}-\nu_{\mathrm{V}}=$ integer which simply transfer the transverse oscillation energy from one direction to the other in a periodic manner. Under the normal operating condition, there is at present no evidence that the coupling is causing any problem. Thus it must be admitted that the continuing effort of the main ring group to reduce the coupling is partially for an esthetic reason; people naturally want the ring to be "clean". Lest this statement should lead to some misunderstandings (and possibly cause a few raised eyebrows as well), one should emphasize that a "clean" accelerator is usually much better than a "dirty" one for reasons which are not always so obvious. More logical argument may cite an increase of the vertical emittance, which may induce the beam loss during the resonant extraction when the horizontal excursion of the beam is necessarily large. There is still a possibility (remote though it might be) that the multi-turn injection into the booster and even a two-turn transverse stacking in the main ring may regain their lost popularities. At the least, the coupling is a real nuisance when one wants to measure the tunes accurately. ${ }^{1}$ This will become increasingly important when the main ring must be operated as a storage ring in the not-too-distant future.

Two methods have been in use in the main ring to reduce the amount of linear coupling, one mostly for the injection and the other for high field. There are eighteeen correction skew quadru- 
poles distributed around the ring, three in each sector at stations \#27, \#37 and \#43 which are all vertical stations (that is, $\beta_{V} \simeq 95 \mathrm{~m}$ and $\left.\beta_{H} \simeq 35 \mathrm{~m}\right)$. In order to control linear coupling resonances of the type $\nu_{\mathrm{H}} \pm \nu_{\mathrm{V}}=\mathrm{N}(\neq 0)$ each element can be excited independently. The maximum current is $\pm 5 \mathrm{~A}$ (not pulsed) and the gradient length is

$$
\int\left(\partial B_{x} / \partial x\right) d l=-\int\left(\partial B_{y} / \partial y\right) d l=0.064 \mathrm{~kg} /+1 A
$$

It is convenient to define the quantity

$$
Q_{\mathbf{s}} \equiv I \mathrm{dl}\left(\partial \mathrm{B}_{\mathrm{x}} / \partial \mathrm{x}\right) /(\mathrm{B} \rho)
$$

where $(B \rho)$ in $\mathrm{kG}-\mathrm{m}=$ (beam momentum in $\mathrm{GeV} / \mathrm{C}$ )/0.02998. The $\operatorname{maxi-}$ mum amount of $Q_{s}$ one can get from the correction system is then

$$
\begin{aligned}
Q_{S} & = \pm(18 \times 5 A \times .064 \mathrm{kG} / \mathrm{A}) /(\mathrm{B} \rho) \\
& = \pm 1.94 \times 10^{-2} \mathrm{~m}^{-1} \text { at injection }
\end{aligned}
$$

At higher momenta, this is not large enough to compensate for the skew quadrupole field in the ring and twelve 7 ' regular quadrupoles are rolled to create the necessary skew field. This roll is commonly known as the "Edwards-stiening Roll".

In 1972 Rae stiening made a series of coupling measurements at $169 \mathrm{GeV}$ and the data were analyzed by Don Edwards. ${ }^{2}$ In order to reduce the amount of coupling, they rolled quadrupoles at vertical stations \#25 and \#43, each by 8 mrad. $^{3}$ The direction of the roll is "wall-side" down and this corresponds to

$$
Q_{S}=-7.37 \times 10^{-3} \mathrm{~m}^{-1} \text { for } 96 \mathrm{mrad} \text {. }
$$

Possible causes of the skew quadrupole field in the main ring have been explained by Lee Teng in his TM report ${ }^{4}$ which also contains a 
simplified analytical treatment of the coupling. One of the findings by Edwards and Stiening is that the coupling behavior is consistent with the average skew quadrupole field in the ring and this field seems to be distributed all around the ring. They could not detect any localized sudden increase of the coupling.

The interest in the subject was aroused in late 1976 when all dipoles were rolled to the proper position. A dipole roll usually makes a change in the average position of the vertical closed orbit. This is coupled with the sextupole field in dipoles to create a skew quadrupole field. 4 Indeed measurements at $290 \mathrm{GeV}$ before and after the dipole roll revealed that the value of $Q_{S}$ in the ring increased from $(1 \sim 2) \times 10^{-3} \mathrm{~m}^{-1}$ to $(2.5 \sim 3.5) \times 10^{-3} \mathrm{~m}^{-1}$. There were complaints from the switchyard group on the increased extraction beam loss. The subsequent measurement in May 1977 was somewhat ambiguous but the result was not in disagreement with $Q \simeq 3 \times 10^{-3} \mathrm{~m}^{-1}$. It was then decided to roll the same twelve quadrupoles by $2.5 \mathrm{mrad}$ each (corresponding to $Q_{S}=-2.3 \times 10^{-3} \mathrm{~m}^{-1}$ ) and the roll of six quadrupoles at stations \#25 (total $13.6 \mathrm{mrad}$ ) was made on July 5-7. Unfortunately, the confusion regarding the sign of the horizontal beam excursion on page 47 of the applications program resulted in the wrong choice of roll direction. As a consequence, the value of $Q_{S}$ was further increased to $(3 \sim 4) \times 10^{-3} \mathrm{~m}^{-1}$. The corrective measure was taken in September by Roy Rubinstein who also measured the roll angle of all quadrupoles. The following is presumably the true status of the present main ring (plus sign indicates "wall-side" down):

Stations \#25 and \#43

All other vertical stations

All horizontal stations
$+126.3 \mathrm{mrad}$

$-\quad 7.7 \mathrm{mrad}$

$-\quad 3.8 \mathrm{mrad}$ 
(In computing these numbers, roll angles of short quadrupoles are multiplied by $1.32 / 2.13$.

Measured value of $Q_{\mathrm{S}}$ (September and December) $=+(1.3 \sim 1.4) \times 10^{-3} \mathrm{~m}^{-1}$. From this, one would surmise that the total skew quadrupole field in the ring at high field was, before any correction.

$$
Q_{S}=+1.1 \times 10^{-2} \mathrm{~m}^{-1}
$$

The present value of $Q_{s}$ suggests a further roll of $18 \mathrm{mrad}(1.5$ mrad each at \#25 and \#43) in the same direction. The total roll of each quadrupole at \#25 and \#43 will then be $+12 \mathrm{mrad}$ of which 8 mrad is the original Edwards-Stiening roll.

II.

Since rolling a quadrupole is always a painful undertaking, it may be wise to review the problem again in order to avoid any pitfalls in the project. The main purpose of this note is to point out that a further roll of the quadrupoles may not reduce the amount of coupling substantially. There is an indirect evidence to support this contention. Stan Pruss has found out that, even with the full range of $\pm 5 \mathrm{~A}$, one cannot eliminate the coupling completely at $8 \mathrm{GeV}$ using the correction skew quadrupoles. It is of course possible (but unlikely) that the remanent skew field is stronger than what the correction system can handle. One should really confirm that, by changing the current from $+5 \mathrm{~A}$ to $-5 \mathrm{~A}$, the quantity $Q_{\mathbf{s}}$ changes its sign from positive to negative. This can be seen from the change of pattern in the induced vertical oscillation when the beam is horizontally kicked by the pinger at E48. For example, with $\nu_{\mathrm{H}}=19.42 \sim 19.43$ and $\nu_{\mathrm{V}}=19.38 \sim 19.39$, a horizontal kick will produce on page 47 of the console program 


\begin{tabular}{|c|c|c|c|}
\hline & $Q_{S}$ positive & & \\
\hline Turn number & $x$ at $\mathrm{E} 46$ & turn number & $y$ at $\mathrm{E} 49$ \\
\hline 4 & large positive & 8 & positive \\
\hline 5 & large negative & 9 & large negative \\
\hline 6 & small positive & 10 & large positive \\
\hline
\end{tabular}

When $Q_{S}$ is changed to negative, the vertical pattern gets reversed with the same horizontal pattern.

Theoretically, it is entirely possible that the coupling does not vanish as $Q_{s}$ goes through zero. When the beam is kicked horizontally, the maximum fraction of the energy that can be transferred to the vertical direction is 1,5

$$
\left(2\left|c_{0}\right|\right)^{2} /\left[\Delta^{2}+\left(2\left|c_{0}\right|\right)^{2}\right]
$$

where

$$
\Delta \equiv \nu_{H}-v_{V}
$$

and (s $\equiv$ distance along the central orbit)

$$
\begin{aligned}
c_{0} \equiv & k_{1}+i k_{2} \\
= & (1 / 4 \pi) \text { o ds }\left(\beta_{H^{\beta}}\right)^{1 / 2}\left(\partial B_{x} / \partial x\right)(B \rho)^{-1} e^{i\left(\theta_{x}-\theta_{y}\right)} \\
& \theta_{x(y)} \text { at } s=\int_{0}^{s} d s / \beta_{H(V)}-\nu_{H(V)} \theta \\
& (\theta \equiv s / \text { average radius of the ring) }
\end{aligned}
$$

In analyzing the data on coupling, ${ }^{1}$ it has always been assumed that the complex parameter $c_{0}$ can be replaced by a real parameter

$$
k=(1 / 4 \pi) \oint\left(\beta_{H} \beta_{V}\right)^{1 / 2}\left(\partial B_{x} / \partial x\right) /(B \rho) d s
$$


Except in the long-straight insertions, $\left(\beta_{\mathrm{H}} \beta_{\mathrm{V}}\right)^{1 / 2} \simeq 53 \mathrm{~m}$ and

$$
\mathrm{k} \simeq 4.2 \mathrm{~m} \times \mathrm{Q}_{\mathrm{S}}
$$

In this approximation, the coupling will vanish when $Q_{S}$ is zero. on the other hand, the more rigorous treatment with $c_{0}$ shows that a complete elimination of the coupling requires both $k_{1}$ and $k_{2}$ to be zero. The defect of the present correction scheme in the main ring is that the quantity $\left(\theta_{X}-\theta_{Y}\right)$ takes almost the same value at all stations where quadrupoles are rolled or correction elements exist. Consequently, it is not possible to make both $k_{1}$ and $k_{2}$ vanish simultaneously.

Because of the six-fold symmetry of the main ring lattice, both $\theta_{\mathrm{x}}$ and $\theta_{\mathrm{y}}$ are periodic with the same periodicity six. For $\left|\nu_{\mathrm{H}}-\nu_{\mathrm{V}}\right|<0.05$, the contribution from $\left(\nu_{\mathrm{H}}-\nu_{\mathrm{V}}\right) \theta$ in $\left(\theta_{\mathrm{X}}-\theta_{\mathrm{Y}}\right)$ is less than $0.05 \times 60^{\circ}=3^{\circ}$ and

$$
\theta_{\mathrm{x}}-\theta_{\mathrm{y}} \simeq \int \mathrm{ds} / \beta_{\mathrm{H}}-\int \mathrm{ds} / \beta_{\mathrm{V}}
$$

The choice of the origin $s=0(\theta=0)$ is of course immaterial and the only relevant quantity is the variation of $\left(\theta_{x}-\theta_{y}\right)$ as a function of $\mathrm{s}$. When the origin is chosen to be at the center of one of the horizontal quadrupoles, $\left(\theta_{x}-\theta_{y}\right)$ is negative from there to the next (vertical) quadrupole and positive in the next half cell. The pattern repeats in succeeding cells but changes significantly in the long straight insertions. This is shown in Fig. 1. With this choice of the origin, one can never modify $k_{2}$ (imaginary part of $c_{0}$ ) using either rolled quadrupoles or correction elements since $\left(\theta_{\mathrm{x}}-\right.$ $\left.\theta_{y}\right) \simeq 0$ at all stations (except at \#49 and \#11). How much one can accomplish with the present correction scheme depends entirely on 
the magnitude of $k_{2}$ which exists in the ring; a complete elimination of coupling is possible only if $\left|k_{2}\right|$ happens to be very small.

\section{III.}

It has been pointed out in the appendix of reference 1 that, in principle, one can gain information on the imaginary part of $\mathrm{c}_{0}$ by including the parameter $\delta \equiv \tan ^{-1}\left(k_{2} / k_{1}\right)$ in the analysis of coupling data. However, this requires a very accurate measurement of the induced vertical oscillation. A more orthodox way ${ }^{5}$ is to kick the beam in the direction $d z / d x=\sqrt{\beta_{H} / \beta}$. The shift of the amplitude modulation from what follows after a simple horizontal kick $(\mathrm{dz} / \mathrm{dx}=0)$ is related to the ratio $\left(\mathrm{k}_{2} / \mathrm{k}_{1}\right)$.

A simple but reasonable model, with a thin-lens skew quadrupole field of the identical strength at the end of each quadrupole, has been used in the following examples which illustrate the importance of controlling both $k_{1}$ and $k_{2}$. The integrated total strength of the skew field in the ring is assumed to be $Q_{S}=+1.1 \times 10^{-2} \mathrm{~m}^{-1}$. Case A

Quadrupoles at stations \#25 and \#43 are rolled by +10 mrad each and the current of all correction skew quadrupoles at \#27, \#37 and \#43 is varied such that the combined skew field strength corresponds to the value of $Q_{S}$ from $-2.7 \times 10^{-3} \mathrm{~m}^{-1}$ to $+3.35 \times 10^{-3} \mathrm{~m}^{-1}$. For each $Q_{S}$, the beam is kicked horizontally at $E 48$ (initial condition $\mathrm{x}=\mathrm{y}=\mathrm{dy} / \mathrm{ds}=0, \mathrm{dx} / \mathrm{ds} \neq 0)$. Thereafter, transverse motions are numerically traced for 100 revolutions. Fig.2 shows the maximum vertical excursion of the beam at $\mathrm{E} 49$ in arbitrary units as a function of $Q_{S}$. The amplitude takes the smallest value near $Q_{S}=0$ but it is not significantly less than the amplitude at $Q_{S}=+\left(1.3^{\sim} 1.4\right) \times$ 
$10^{-3} \mathrm{~m}^{-1}$ (indicated by arrows in the figure) which is the measured value of $Q_{S}$ in the present ring. For $Q_{S}=0, F i g .3$ is a simulation of what one would see on page 47 of the console program where the vertical scale is believed to be $2 / 3$ of the horizontal scale. 6 Case B

Correction skew quadrupoles are placed at stations \#11 and \#49 instead of \#27, \#37, and \#43. If the roll angle of quadrupoles at \#25 and \#43 is taken to be $9.6 \mathrm{mrad}$ each, the ratio $\mathrm{k}_{2} / \mathrm{k}_{1}$ is constant as the current of the correction elements is varied. Fig. 4 is similar to Fig. 2 but the induced vertical amplitude for $\mathrm{k}_{1}=$ $\mathrm{k}_{2}=0$ is smaller by an order of magnitude compared to the amplitude for $Q_{S}=0$. It should be emphasized that there are only twelve correction elements in this case compared to eighteen in case A. clearly one does not get a better compensation by merely increasing the number of correction elements. What is essential is the simultaneous control of $\mathrm{k}_{1}$ and $\mathrm{k}_{2}$.

IV.

Although it is reasonable, the model used here is after all just a model and not the real picture of the main ring. For example, it is almost impossible to have skew field in the ring with exact six-fold symmetry. The effect of the 39 th harmonic component, which does not exist in the model, could be equally important for the coupling because of the contribution from the resonance $\nu_{\mathrm{H}}+\nu_{\mathrm{V}}=39$. One must then control another complex quantity like $c_{0}$ (altogether four parameters ${ }^{7}$ ) but with the phase $\theta_{x}+\theta_{y}-39 \theta$. At high field, one may have to roll quadrupoles at stations \#ll and \#49 but the amount of roll is an unknown quantity unless a good measurement of 
$\mathrm{k}_{2}$ is made. Besides, rolling quadrupoles in the transfer hall is a dangerous game to play. For the injection, the future program may proceed as follows:

1. Establish that $Q_{S}$ changes its sign when the current of correction elements is varied from $+5 \mathrm{~A}$ to $-5 \mathrm{~A}$.

2. Find out whether the 39th harmonic component created by correction elements affects the amount of coupling.

3. Install correction skew quadrupoles at \#11 and \#49.

The project is somewhat academic at present inasmuch as there is no obvious reason to believe that the reduction of the coupling during injection will improve the performance of the main ring.

Discussions with the members of the main ring group have been invaluable in preparing this note and I am grateful to them for providing me with coupling data.

\section{References}

1. EXP-85, September 14, 1977.

2. EXP-27, November 8, 1972 .

3. The amount of roll will be the same, with the opposite direction, if quadrupoles at horizontal stations are to be rolled.

4. L.C. Teng, TM-382, July 21, 1972.

5. G. Guinard, Selection of Formulae Concerning Proton Storage Rings, CERN 77-10, 6 June 1977, p. 63 .

6. Frank Turkot, private communication.

7. S. Ohnuma, TM-393, September 25, 1972. 
-1
$ت$
0
0
0
$\pi$
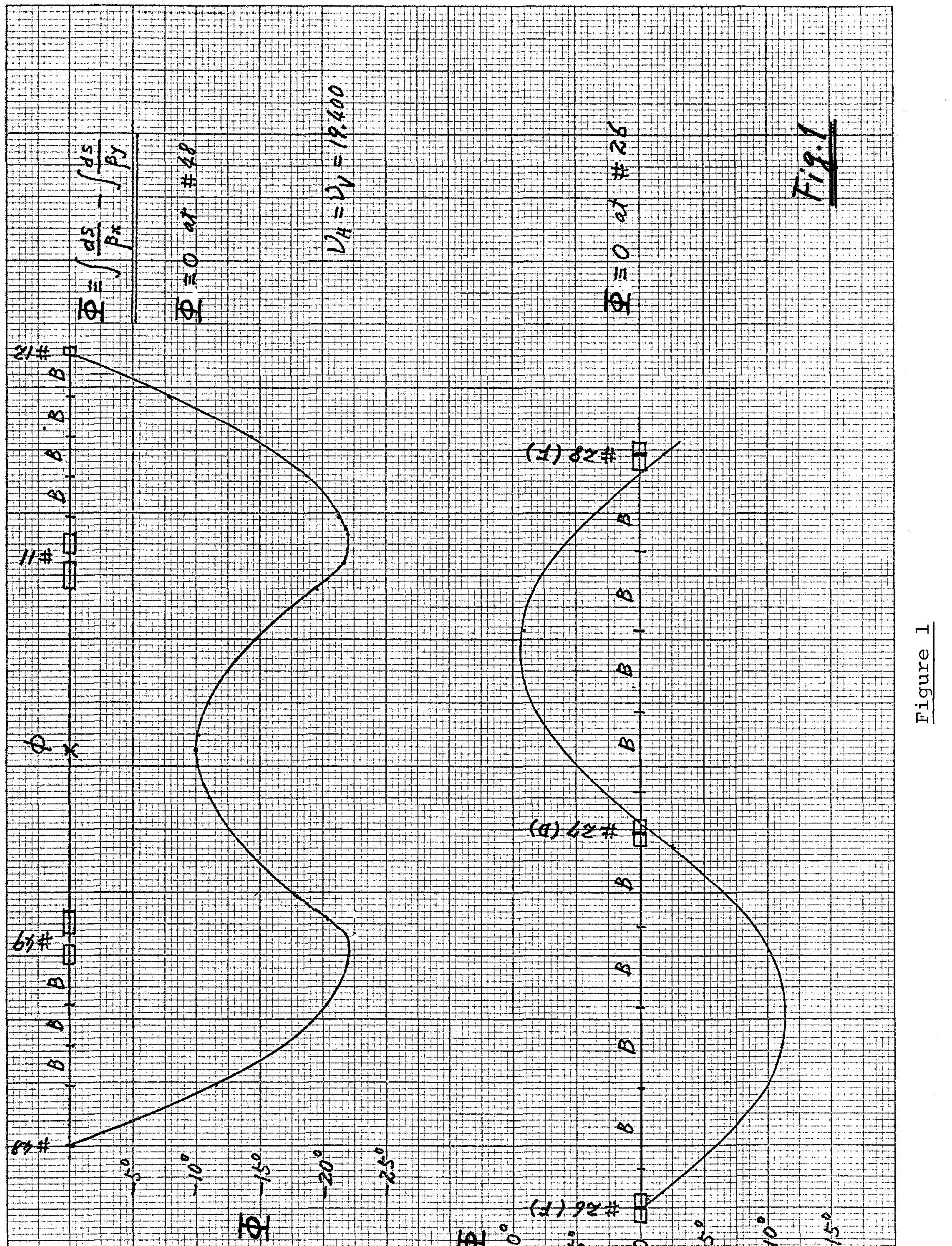


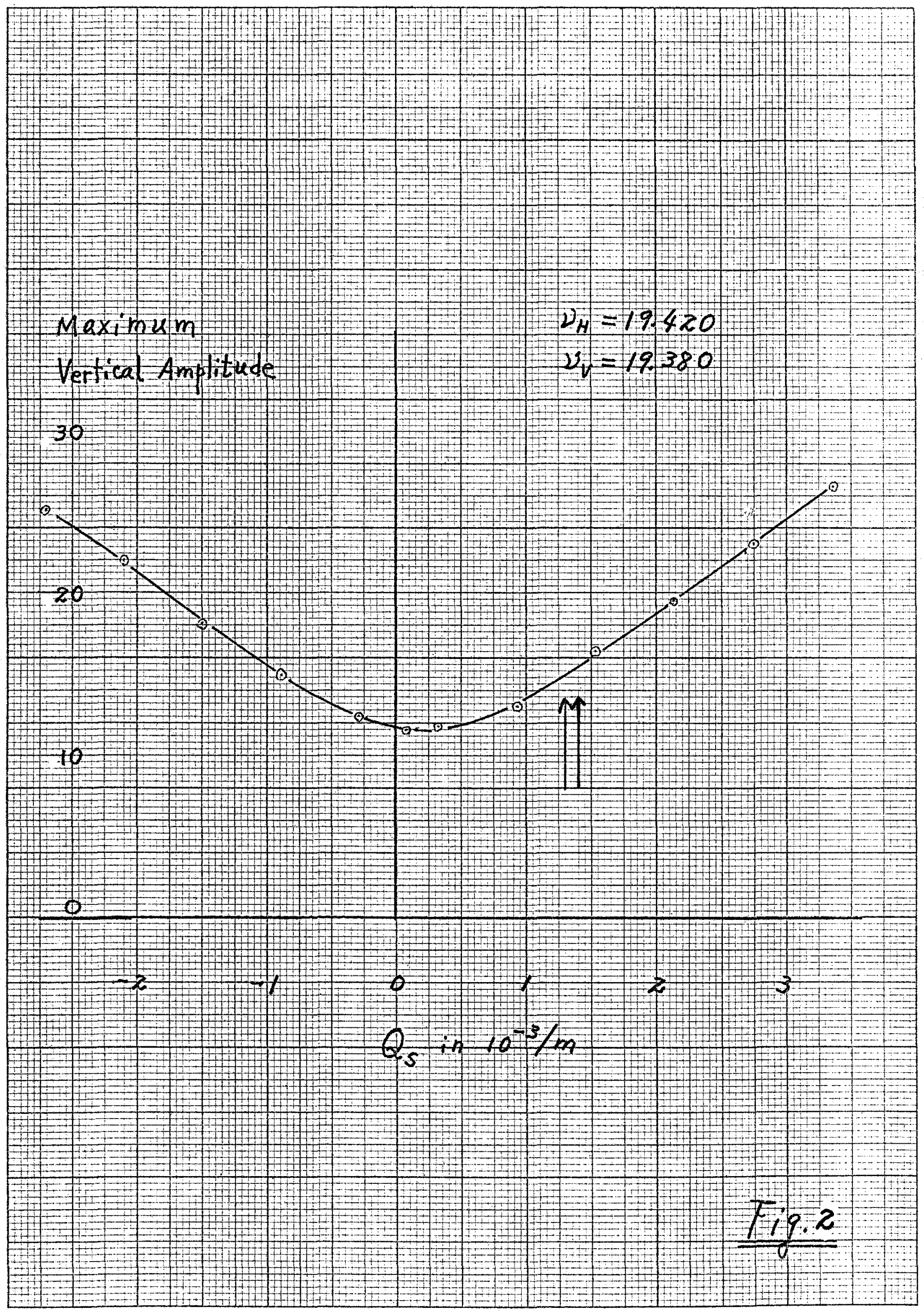




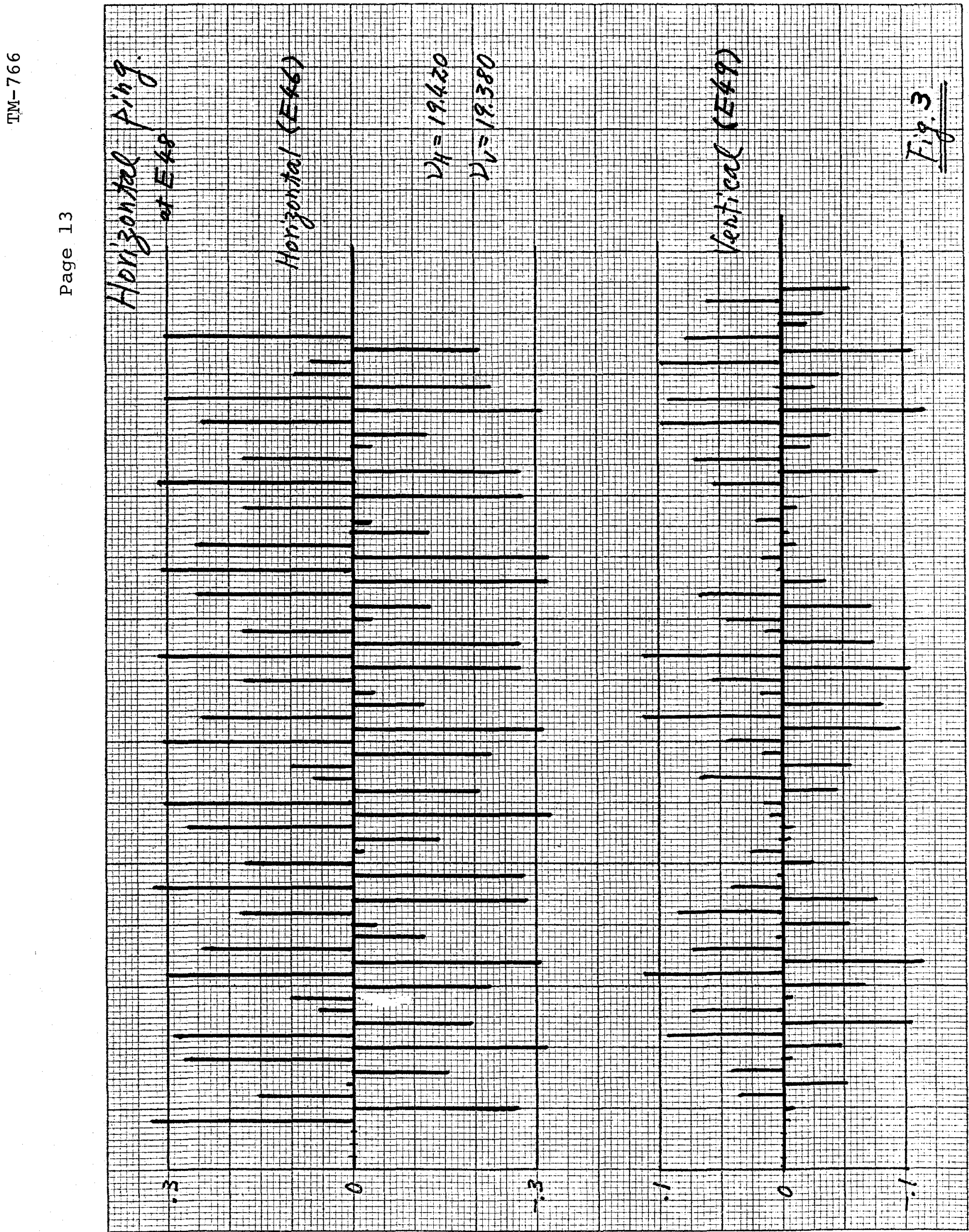

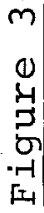




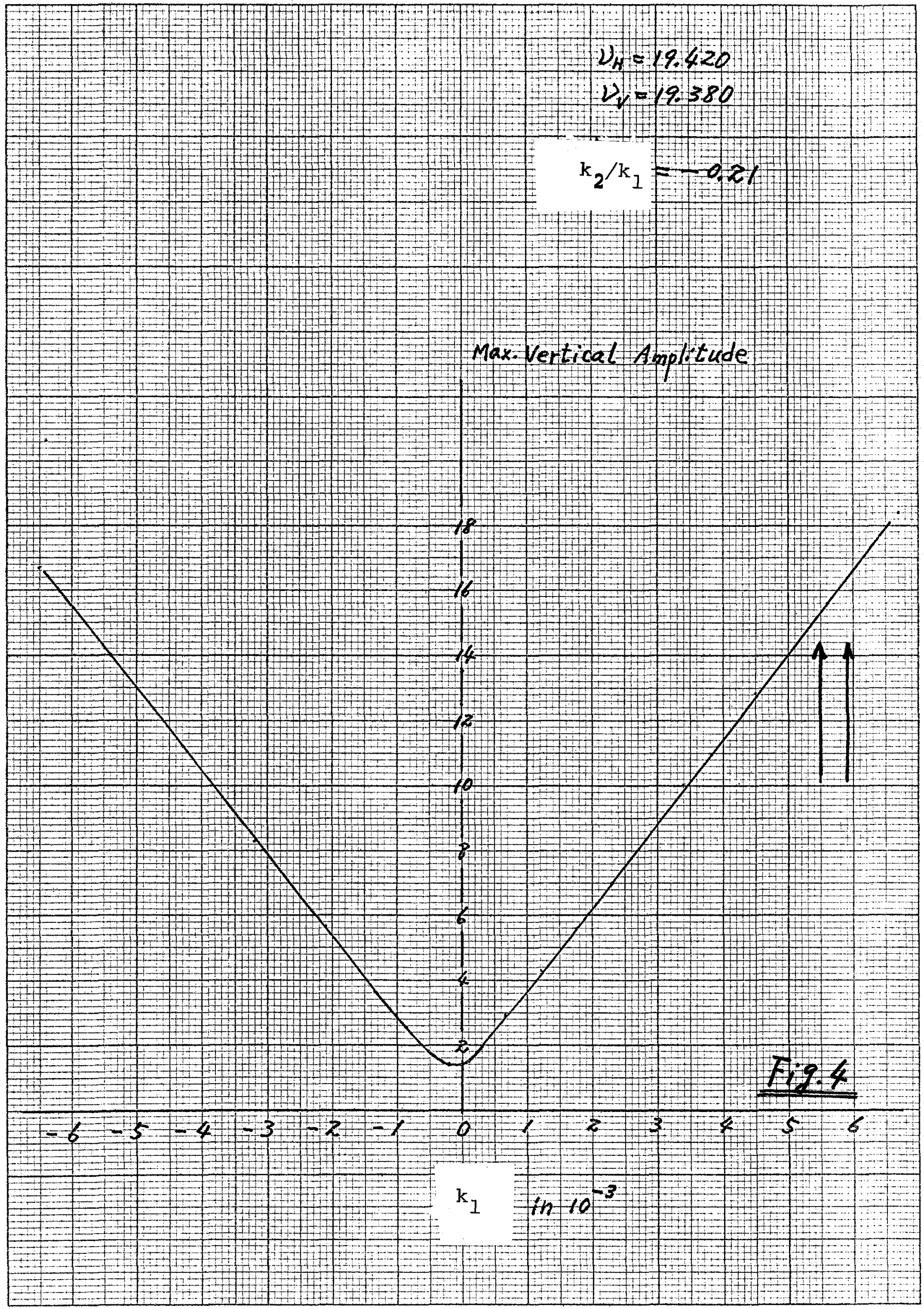

